\title{
Dynamic Hippocampal Circuits Support Learning- and Memory-Guided Behaviors
}

\author{
Emily B. Anderson, Irene Grossrubatscher, and Loren Frank \\ Department of Physiology and Center for Integrative Neuroscience, University of California, \\ San Francisco, San Francisco, California 94143 \\ Correspondence: loren@phy.ucsf.edu
}

\begin{abstract}
The ability to learn spatial relationships and to modify stored representations when the world changes is essential for survival. In recent years, our understanding of the neural activity that underlie these learning and memory processes has improved considerably, in large part to an increase in the number of studies that use electrophysiological recordings as animals learn about their environments. In this review, we summarize some key findings from our laboratory, with a focus on recent discoveries that indicate that the rat hippocampus supports learning and decision-making behaviors via dynamic and smooth transitions in neural representation, internal processing state, and coupling with related brain structures.
\end{abstract}

Our memories are central to guiding our decisions and defining us as individuals. Historically, there have been two distinct approaches toward studying memory and the brain structures that support it: a cognitive approach and an electrophysiological approach. The first approach, perhaps best known for the seminal studies on patient H.M. (Scoville and Milner 1957; Squire and Bayley 2007; Eichenbaum 2013) uses a combination of lesion, fMRI, and behavioral methods to examine the role of different brain structures in specific learning and memory tasks. A key insight from this approach is that different brain circuits support different types of memory (Eichenbaum and Cohen 2001). From these studies, a particular region of the brain, the hippocampus (see Box 1), has emerged as particularly important in encoding episodic or declarative memories - that is, the memories of distinct, autobiographical experiences that are essential to our sense of self.

To gain insight into the specific neural mechanisms underlying memory, electrophysiologists place recording electrodes within the hippocampus and connected structures to examine how the electrical signals of individual neurons change as animals engage in tasks. Although technically challenging, the electrophysiological approach offers greater temporal precision and improved ability to identify individual circuit components. Historically, however, most electrophysiological studies of the hippocampus did not focus on learning and memory behaviors, perhaps because most classic memory tasks lack the repetitive trial structure necessary for neurophysiological data collection (Kim and Frank 2009). Instead, most electrophysiological studies have focused on characterizing the properties of individual "place cells" - hippocampal neurons that elicit action potentials at selective locations of an animal's environment - and the role of these cells in constructing an allocentric spatial map of the environment (O'Keefe and Dostrovsky 1971; Best et al. 2001). Consequently, until recently, relatively little was known about how activity at the single-neuron level could support the learning and memory functions of the hippocampus. The question of how single neurons and circuits of the hippocampus support cognitive functions like learning and memory is the main motivator for our work and the focus of this review.

\section{ROLE OF HIPPOCAMPUS IN LEARNING}

Learning is not just the formation of new memories but also a change in representation of the world. Classically, the hippocampus and neocortex had been thought to operate complementary learning systems that divide these tasks (Marr 1971; Buzsáki 1989; McClelland et al. 1995; Sutherland and McNaughton 2000; O'Reilly and Norman 2002). In this view, the hippocampus was thought to form distinct ("pattern separated") representations of the world during an initial experience. These patterns would then be essentially static within the hippocampal circuit. Once these memories were encoded by the hippocampus, reactivation of those memories during sleep was thought to drive consolidation in cortical circuits (Wilson and McNaughton 1994; Buzsáki 1996; O’Neill et al. 2010). This reactivation occurs during "sharp-wave ripple" (SWR) events, which manifest as bursts of high frequency $(150-250-\mathrm{Hz})$ power in the local-field potential recorded in area CA1 (Buzsáki 1986). The neocortex would then learn slowly about the statistical regularities of the environment as the hippocampus repeatedly presented these memory sequences (i.e., replay events) during SWRs occurring in slow-wave sleep. Thus, the hippocampus was thought to represent the world while animals are awake and to send information to the neocortex for consolidation during sleep.

Although this theoretical framework has proven influential, it was developed at a time when most studies of 


\section{BOX 1: THE HIPPOCAMPAL CIRCUIT}

The rodent hippocampus is a pair of banana-shaped structures in the medial temporal lobe (Fig. 1). As with many brain regions, the hippocampus is highly interconnected (Amaral and Witter 1995). These connections include a large number of feed-forward connections, which begin with a projection from the entorhinal cortex to all of the hippocampal subdivisions. Information also propagates along multiple internal pathways, including the Mossy fibers of the dentate gyrus that project to areas CA2 and CA3, and the Schäffer collaterals from area CA3 to area CA1. In addition, there are a number of recurrent networks within the hippocampal circuit, including one in the dentate gyrus/hilar region (not shown), a strong CA3 recurrent network, and a recently discovered CA1 recurrent network (Yang et al. 2014).

The hippocampal regions, which differ in their connectivity with subcortical structures (Amaral and Witter 1995), also vary along the dorsoventral axis. Most studies of the rodent hippocampus have focused on the more physically accessible dorsal region, which is noted for neurons that represent specific locations in space ("place cells") and is thought to be important for spatial navigation and memories involving spatial context. In contrast, the less-studied intermediate and ventral hippocampus may play an important role in anxiety and emotional memories (Fanselow and Dong 2010).
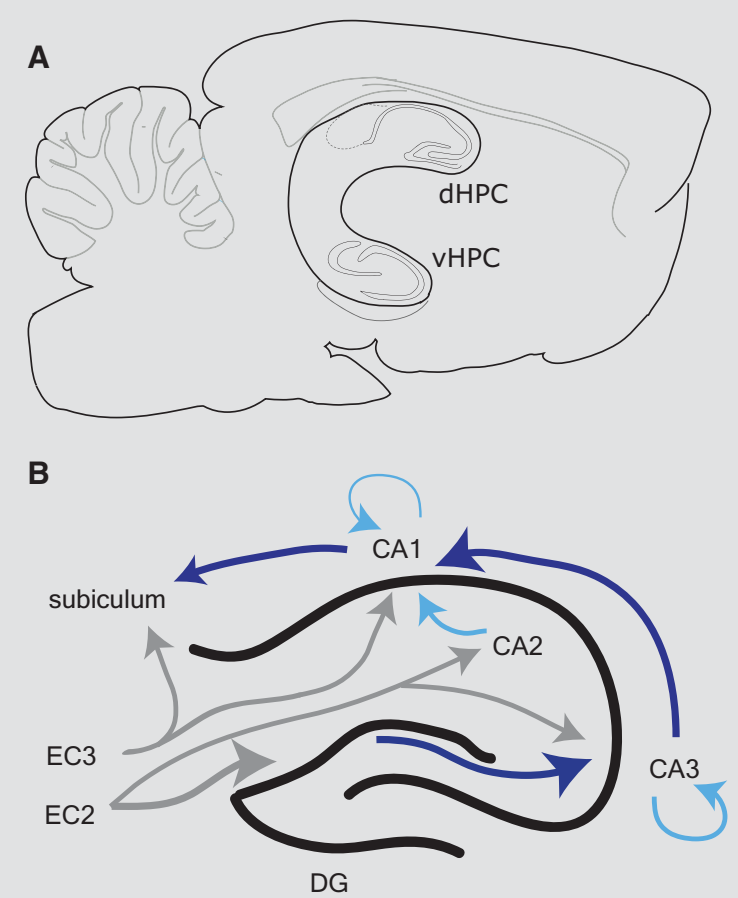

Figure 1. Hippocampal circuit. (A) A sagittal view of the rodent hippocampus, showing the relative locations of the dorsal (dHPC) and ventral (vHPC) hippocampus. (B) Simplified hippocampal circuit diagram. DG: dentate gyrus; EC2 and EC3: layers 2 and 3 of the entorhinal cortex. Outputs from CA1 and the subiculum target the deep layers of the entorhinal cortex as well as many other cortical and subcortical regions (not shown). learning did not use electrophysiological techniques and therefore did not provide data on how learning influences the formation and maintenance of place fields, replay/ reactivation events, or other aspects of hippocampal activity. Similarly, most electrophysiological studies had focused on describing patterns of activity obtained during well-learned tasks performed in familiar environments and thus also did not provide data on how patterns of neural activity support learning. In the following sections, we offer two themes that challenge the classic view and expand the hippocampus's role in learning. First, we discuss results indicating that the hippocampus does not simply form a new and stable representation following an experience. Instead, the hippocampus changes its signaling patterns as environments and objects become more familiar (Cheng and Frank 2008; Karlsson and Frank 2008; Larkin et al. 2014) and as animals learn specific tasks (Dupret et al. 2010; Singer et al. 2010). Second, we describe our findings that the memory replay events that had previously been attributed to slow-wave sleep also occur in the awake state, particularly in the context of new experiences (Cheng and Frank 2008; Karlsson and Frank 2009). Further, we discuss the role of these events in learning- and memory-guided decision-making (Jadhav et al. 2012; Yu and Frank 2014).

\section{HIPPOCAMPAL REPRESENTATIONS CHANGE WITH EXPERIENCE}

We examined the effect of novelty on place cell representations and found that the introduction of novel environments and contexts caused a novelty-dependent increase in place cell firing rate in area CA1 (Karlsson and Frank 2008; Larkin et al. 2014). This increase in firing rate was specific to CA1, as firing rates in CA3 remained stable (Karlsson and Frank 2008; Larkin et al. 2014). Over time, as the environment became more familiar and task performance improved, the distribution of firing rates across the CA1 place cell population spread: High firing rate neurons increased their firing rate, whereas low firing rate neurons decreased in rate (Karlsson and Frank 2008). The result of these changes is that the active CA1 population comes to consist of a relatively small group of cells with strong spatial tuning, creating a sparse, spatially informative population of neurons. Interestingly, we found that as this information con- 
tinues from CA1 to subicular output circuits, the code is further transformed from a sparse code to a highly distributed code that may be more effective for communication with other brain regions (Kim et al. 2012).

These results complemented previous studies showing that the hippocampus is necessary for behaviors associated with contextual novelty. Measurements of evoked response potentials (ERPs) and of the BOLD response in fMRI scans have also shown an increase in activity in the hippocampus at the onset of novel stimuli (Knight 1996; Knight and Nakada 1998). These results led to the proposal that hippocampal area CA1 serves as a novelty detector (Lisman and Otmakhova 2001), as it could compare stored representations in CA3 with sensory information from the entorhinal cortex (see Box 1). Thus, we hypothesize that the increase in firing rates seen in CA1 but not upstream in CA3 could be the physiological mechanism that explains the previously observed hippocampal responses to novelty.

In a related study, we observed other long timescale patterns of change in hippocampal representations. We found that as animals learned specific behavioral tasks, coordinated hippocampal ensembles came to encode generalizations across locations (Singer et al. 2010). As the animals gained more experience with the environment, about half of the population of place cells in CA1 and CA3 developed path equivalent firing, with individual neurons firing in multiple locations with similar behavioral contexts. Such path equivalent ensembles are wellsuited to encode similarities among repeating elements and potentially provide a framework for associating specific behaviors with multiple locations (Frank et al. 2000). At the same time, a population of "classic" place cells remained that could maintain specific location information. Taken together, these results argue that place cell activity not only supports the distinct representations proposed by earlier theories but also more generalized information that can support learned behaviors. We suggest that path equivalent ensembles reflect the representation of common features and behavioral associations across locations.

\section{LEARNING MODULATES HIPPOCAMPAL REACTIVATION}

In addition to these changes in hippocampal representations, we wondered whether learning modulated hippocampal communication to other brain structures. In particular, we were interested in SWR events as these are known to propagate from the hippocampus to cortical structures (Chrobak and Buzsáki 1994; Buzsáki 1996; Csicsvari et al. 2000) and are associated with a timecompressed reactivation of firing patterns associated with previous (Pavlides and Winson 1989; Wilson and McNaughton 1994; Kudrimoti et al. 1999; Nadasdy et al. 1999; Lee and Wilson 2002; Ji and Wilson 2007; O’Neill et al. 2008; Karlsson and Frank 2009) and current (Foster and Wilson 2006; Csicsvari et al. 2007; Diba and Buzsáki 2007; Carr et al. 2011) experiences. Because spiking during these ripple events is phase locked to fast oscillations similar to those used to induce LTP (Buzsáki 1986; Wilson and McNaughton 1994; Sutherland and McNaughton 2000), ripple reactivation has been proposed to drive consolidation of previously formed memories. Interestingly, we found that novelty did in fact modulate this communication: In rest sessions following exposure to a novel environment, there was an increase in the mean firing rate during SWR events (Karlsson and Frank 2008). This finding complemented parallel results from the Csicsvari laboratory (O’Neill et al. 2008). We also observed that novelty increased the correlation of neurons in the rest box with their activity in the previous behavioral session (Karlsson and Frank 2008) suggesting that novel experiences may be more faithfully consolidated during subsequent sleep sessions.

\section{AWAKE REPLAY DURING LEARNING}

Classically, reactivation of past experiences was thought to only occur during sleep, consistent with the idea of sleep as a privileged state for memory consolidation processes (Wilson and McNaughton 1994; Buzsáki 1996; O’Neill et al. 2010). Later, when sequences of place cell activity during SWRs were discovered during awake quiescence (Kudrimoti et al. 1999; Csicsvari et al. 2007 \#1415; Foster and Wilson 2006; Diba and Buzsáki 2007), it was thought that although replay during sleep involved the reactivation of stored representations in the absence of specific sensory input, awake replay depended on sensory input from the current environment.

Recently, however, we discovered robust remote replay of past experiences during waking behavior (Karlsson and Frank 2009). Indeed, awake replay of a remote environment is typically a higher fidelity recapitulation of past experiences than replay during quiescence or sleep (Karlsson and Frank 2009). This led us to ask whether awake replay events might play an important role in memory retrieval and consolidation as animals learn about new environments and tasks (Carr et al. 2011).

Consistent with this hypothesis, we found an increase in the number of awake SWR events when animals were exposed to novel environments (Cheng and Frank 2008) or novel contexts (Larkin et al. 2014). We also found that novelty increased the firing rate of CA1 neurons awake during SWRs and increased the degree of coordination of neurons representing novel experiences during SWRs (Cheng and Frank 2008; Larkin et al. 2014). This suggests that in a similar fashion to SWRs occurring during sleep, awake SWRs more faithfully reflect novel experiences than familiar ones. This increase in firing rate and coordination associated with novelty was specific to activity during ripples in CA1, as no changes were seen in CA3 (Larkin et al. 2014) or outside ripple events (Cheng and Frank 2008; Larkin et al. 2014).

Parallel studies had shown that SWR events during sleep play an important role in task learning (Girardeau et al. 2009), and that the strength of awake reactivation 
predicts subsequent memory, suggesting that awake SWRs may indeed play an important role in learning (Dupret et al. 2010). We also observe an increase in the number of SWRs following reward, and the content of these ripples tended to reflect paths associated with reward location (Singer and Frank 2009), which could serve as a mechanism to bind rewarding outcomes to preceding experiences.

These studies did not establish a causal role for awake SWRs in learning, however. We therefore developed a real-time SWR detection system and used it to detect and then electrically interrupt awake SWRs as animals learned a hippocampus-dependent spatial alternation task (Jadhav et al. 2012). We found that the disruption of SWRs during learning caused consistent, strong, and significant deficits in performance of the task (Fig. 2). Interestingly, we found that this impairment was selective: Only outbound trials that required integration of information about immediate past and potential future options were impaired (Jadhav et al. 2012). Further, place-field activity was unaffected, indicating that the impairment was specific to the role of SWR events. As we will discuss in the next section, we hypothesize that reactivation of path trajectories during SWRs provides information about past locations and possible future options to guide decisions made in structures such as the prefrontal cortex.

\section{AWAKE REPLAY IN RETRIEVAL, DELIBERATION AND DECISION-MAKING}

We propose that one purpose of awake replay events is to contribute to internal deliberation processes whereby different routes through the animals "cognitive map" are evaluated (Yu and Frank 2014). Consistent with this idea, awake ripples frequently occur when animals pause at choice points (Karlsson and Frank 2009; Jadhav et al. 2012). Further, decoding of place cell reactivation shows that replay trajectories are biased to include the current location of the animal (Davidson et al. 2009; Karlsson and Frank 2009) and, when pertinent, can be biased to terminate at a rewarded goal location (Dupret et al. 2010; Pfeiffer and Foster 2013).

We have also shown that during learning awake SWR reactivation is more coordinated and more intense preceding correct decisions than incorrect ones (Singer et al. 2013), suggesting that replay during SWRs contributes to successful decision-making. Interestingly, however, we found that replay trajectories corresponding to both correct and incorrect choices often occur before a choice. This suggests that processing downstream (perhaps in prefrontal cortex) is critical for evaluating the content of these ripples and in making the ultimate behavioral decision (Singer et al. 2013; Yu and Frank 2014).
A
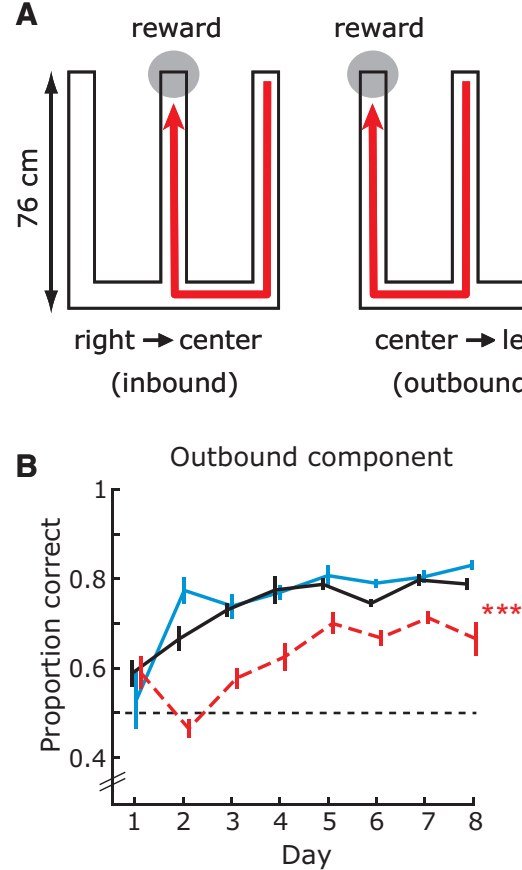

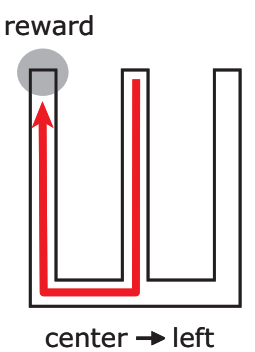

(outbound)

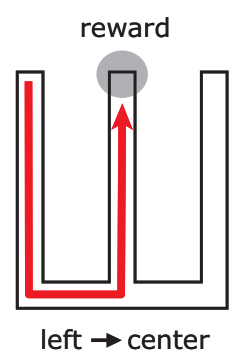

(inbound)

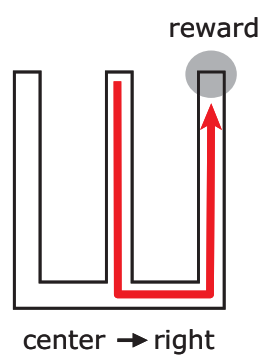

(outbound)

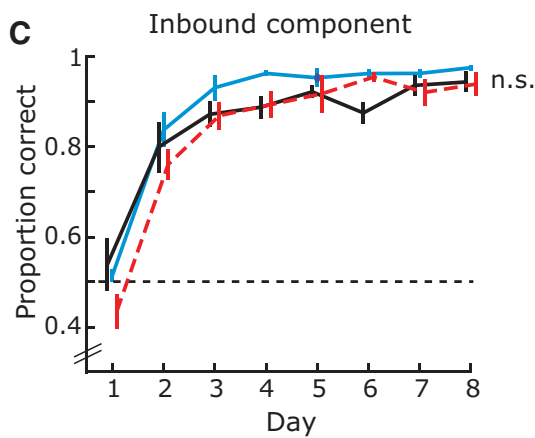

Figure 2. Disruption of SWRs during learning causes strong deficits in task performance. ( $A$ ) Spatial alternation "W-maze" task (Kim and Frank 2009). Rats must learn two rules. When in an outer arm, animals must return to the center arm (inbound component). Thus, this "inbound" trial depends only on the rat's current location and is independent of previous history. In contrast, when leaving the center arm, the animal should not return to the most recently visited arm but instead alternate between the outer arms (outbound component). (B) Performance during the outbound component. Electrical disruption of SWR events significantly impaired performance $(p<0.001)$ compared with control groups. Dashed red line indicates performance with electrical stimulation, black line indicates performance in unstimulated animals, and light blue indicates control stimulation animals. $(C)$ Performance during the inbound component. In contrast to the outbound behavior, disruption of SWRs did not alter performance. Colors indicate performance as in B. (Modified from Jadhav et al. 2012.) 


\section{FROM GEOMETRICAL TO TOPOLOGICAL "COGNITIVE MAP" IN THE HIPPOCAMPUS}

In a parallel line of investigation, we examined the nature of the representation of space in the hippocampus. Place-field activity has often been characterized as representing geometric features about the environment, and the impact of some environmental manipulations can be explained in geometrical terms (O'Keefe and Burgess 1996; Eichenbaum 2000; Fenton et al. 2000a,b). In this view, place-field firing can be explained as a result of synthesizing information about distances and angles to landmarks arising from various sources, including selfmotion and external, sensory cues.

Although a geometrical perspective is appealing, place cells respond to a wide variety of factors that include visual cues, head direction, goal-planning, and so on. It is not immediately clear how all of these factors can be integrated in a geometrical framework. We therefore proposed that place-field activity is better conceived as a topological representation of space. In this view, individual place cells represent regions of space and sets of place cells that fire together represent regions that are connected, but the firing of the cells does not explicitly represent distances or angles (Dabaghian et al. 2012).

We tested this hypothesis by constructing an environment where the topology was held constant while the geometry changed from trial to trial. In this dynamic environment, place-field activity was highly unstable when the specific locations, distances, and angles were considered, but stable when only the connectivity of the space was considered (Dabaghian et al. 2014). Therefore, we suggest that the hippocampal map provides information about the connectivity of spatial environments to downstream brain regions, an idea that translates naturally to the hippocampus providing a representation that would capture the connected elements of experience (Eichenbaum 2000; Dabaghian et al. 2014).

\section{DYNAMIC ROLE FOR HIPPOCAMPUS IN INFORMATION PROCESSING}

We have also examined the dynamics of hippocampal information processing states. The hippocampus is typically described as having two distinct states: one that supports encoding of new memories and a second that consolidates these memories into long-lasting distributed representations (Marr 1971; Buzsáki 1989). During periods of active exploration, processed sensory input drives coordinated neuronal activity and is associated with synchronous oscillations in the $7-10-\mathrm{Hz} \theta$ range (Buzsáki 2002). This "external" drive is mediated by the entorhinal cortex (Box 1) and likely supports the encoding of memories. In contrast, during slow-wave sleep or periods of quiet stillness, the hippocampus is driven by internal inputs, with stored information broadcast out of the hippocampus (via replay events) to consolidate memories in distributed hippocampal-neocortical circuits.

Our results suggest that in contrast to the two-state model, hippocampal activity can be better described as a dy- namic continuum with rapid and smooth transitions in information flow and processing. Such "on the fly" reconfiguration allows the hippocampus to perform different functions at different times, depending on the behavioral state of the animal. Because relatively little was known about how the external entorhinal and the internal CA3 inputs combined to influence CA1 output, we performed a series of experiments to understand how information flow through these pathways varied as a function of behavioral state (Kemere et al. 2013). We first examined changes in $\gamma$ frequencies, as recent studies had shown that power in the $30-50-\mathrm{Hz}$ slow $\gamma$ band of the local-field potential reflects the strength of coupling of CA 3 to CA1 (Csicsvari et al. 2000; Colgin et al. 2009; Nakashiba et al. 2009), whereas power in the $50-100-\mathrm{Hz}$ fast $\gamma$ band reflects the strength of EC3-CA1 coupling (Colgin et al. 2009). Both ranges of $\gamma$ are seen during exploration (Colgin et al. 2009), and we have shown that SWRs and replay events occur during transient increases in slow $\gamma$ power and synchrony across the dorsal CA3 and CA1 networks (Carr and Frank 2012). Further, as the strength of low $\gamma$ power increases, the quality of replay events also improves (Carr and Frank 2012), suggesting that the strength of CA3 input into CA1 modulates replay fidelity. Transient increases in CA3-CA1 coupling have also been seen when animals approached a position in a maze where they needed to make a memory-guided decision (Montgomery and Buzsáki 2007), consistent with the hypothesis that CA3 drive of CA1 is important for online memory retrieval.

We found that slow $\gamma$ and ripple power decreased smoothly as animals moved progressively faster through their environment (Fig. 3A,B), consistent with weaker CA3 drive of CA1 at high speeds. In sharp contrast, fast $\gamma$ power increased with increasing speed, suggesting greater $\mathrm{EC}$ drive of CA1 as animals moved more quickly (Fig. 3C). We also directly examined the propagation of activity from CA3 to CA1 by electrical stimulation and found that the slope of the field potentials recorded in CA1 varied smoothly as a function of the movement speed of the animal (Fig. 1D). These changes occurred on a timescale of $<1$ sec. Taken together, these results suggest a changing balance between the CA3 and EC inputs to CA1, with CA3 inputs becoming relatively less influential and EC inputs becoming relatively more influential as animals moved more quickly.

This shift from CA3 to EC drive also corresponded to a major change in the spiking output of CA1. The spiking of pairs of CA1 neurons with overlapping place fields was highly correlated at low speeds and these correlations decreased threefold with increasing speed. Thus, momentby-moment changes in behavior that are seen as animals explore new places manifest as a transition from CA3driven, highly correlated activity reflecting learned association (as in awake replay of past experience) at slow speeds to EC-driven, more independent representations that reflect sensory inputs available in the current location at high speeds.

How are these changes in network state regulated? Although there are likely multiple factors that sculpt the 
A

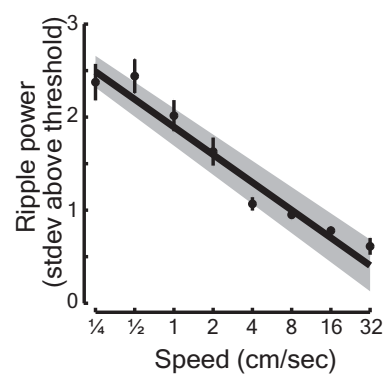

B

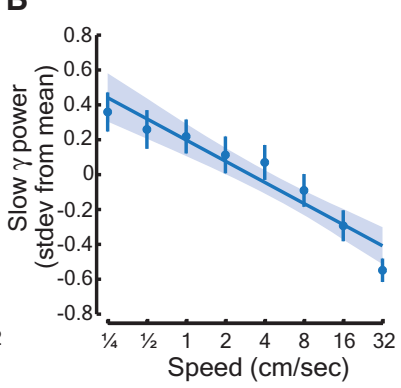

C

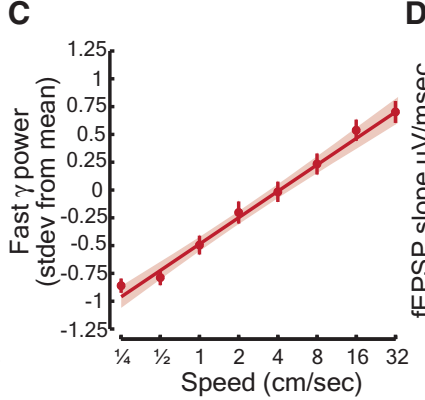

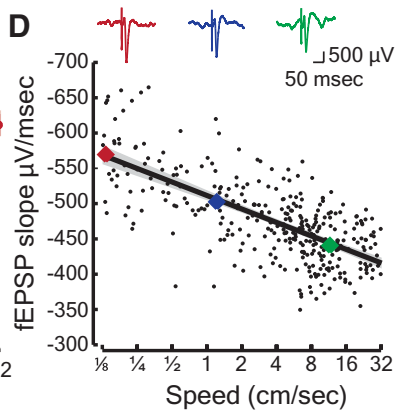

Figure 3. CA1 responses change as function of movement speed. $(A, B)$ Measures related to CA 3 drive of CA1 decline smoothly with increasing movement speed. $(A)$ SWR power; $(B)$ slow $\gamma$ power. Note the log scale for speed. $(C)$ Fast $\gamma$ power, which is related to EC drive of CA1, increases smoothly with movement speed. $(D)$ Linear relationship between the log of movement speed and the slope of evoked field postsynaptic potentials in CA1. Example traces for each colored point shown at right. Over $50 \%$ of the variance in the slope could be explained by the log of speed, indicating that CA1 responses to CA3 stimulation vary closely with movement speed. There was no tendency for variance of our measurements to increase at intermediate speeds as would be expected if the observation of continuous changes was a result of rapid transitions between two distinct states. Error bars represent SEM, shaded areas represent SEM for linear fits. (Modified from Kemere et al. 2013.)

relative strength of these inputs, recent work indicates that changes in cholinergic tone from the medial septum play a critical role in regulating this dynamic balance. Recent experiments have shown that optogenetic stimulation of cholinergic neurons in the medial septum results in a rapid, strong, and significant reduction in hippocampal SWR events in mice (Vandecasteele et al. 2014) and rats (Davidson et al. 2014) that likely reflects a shift away from network activity driven by the CA3-CA1 pathway. This reduction in SWRs appears to be mediated by muscarinic receptors, as application of pilocarpine (a muscarinic receptor agonist) also reduces the frequency of SWRs in vivo (Norimoto et al. 2012). Future work is needed to better understand how cholinergic modulation of hippocampal state is coordinated with other systems (such as septal GABAergic modulation of hippocampal $\theta$ activity) and how changes in hippocampal network activity supports hippocampal function.

\section{CONCLUSION}

In this review, we have highlighted how recording populations of individual hippocampal neurons as animals learn to navigate through an environment to obtain reward has changed our view of how the hippocampus supports learning. Our experiments revealed a strong effect of novelty on hippocampal representations and activity, and showed a causal role for awake SWR events in memory processes. Our work also suggests a topological framework for the hippocampal representation of space, and offers new insights into how the hippocampal circuit supports different information processing functions at different times.

These findings provide a stepping-off point for new studies that seek to understand not just the hippocampus, but the broader hippocampal-cortical and hippocampalsubcortical networks. Given the importance of awake SWRs for memory, we can now ask how these events drive information processing in downstream areas. We can also ask how these events, and network state more generally, are regulated and whether we can manipulate that regulation to alter how the hippocampus processes information. These studies, when combined with continued technology development including high-density recordings and better tools for precisely manipulating neural signals, will drive deeper insight into how interactions between the hippocampus and adjacent structures support learning and memory.

\section{REFERENCES}

Amaral DG, Witter MP. 1995. Hippocampal formation. In The rat nervous system, 2nd ed. (ed. Paxinos G), pp. 443-493. Academic, San Diego.

Best PJ, White AM, Minai A. 2001. Spatial processing in the brain: The activity of hippocampal place cells. Annu Rev Neurosci 24: 459-486.

Buzsáki G. 1986. Hippocampal sharp waves: Their origin and significance. Brain Res 398: 242-252.

Buzsáki G. 1989. Two-stage model of memory trace formation: A role for "noisy" brain states. Neuroscience 31: 551-570.

Buzsáki G. 1996. The hippocampo-neocortical dialogue. Cereb Cortex 6: $81-92$.

Buzsáki G. 2002. Theta oscillations in the hippocampus. Neuron 33: $325-340$.

Carr MF, Frank LM. 2012. A single microcircuit with multiple functions: State dependent information processing in the hippocampus. Curr Opin Neurobiol 22: 704-708.

Carr MF, Jadhav SP, Frank LM. 2011. Hippocampal replay in the awake state: A potential substrate for memory consolidation and retrieval. Nat Neurosci 14: 147-153.

Cheng S, Frank LM. 2008. New experiences enhance coordinated neural activity in the hippocampus. Neuron 57: 303-313.

Chrobak JJ, Buzsáki G. 1994. Selective activation of deep layer (V-VI) retrohippocampal cortical neurons during hippocampal sharp waves in the behaving rat. $J$ Neurosci 14: $6160-$ 6170 .

Colgin LL, Denninger T, Fyhn M, Hafting T, Bonnevie T, Jensen O, Moser MB, Moser EI. 2009. Frequency of $\gamma$ oscillations routes flow of information in the hippocampus. Nature 462: $353-357$.

Csicsvari J, Hirase H, Mamiya A, Buzsáki G. 2000. Ensemble patterns of hippocampal CA3-CA1 neurons during sharp wave-associated population events. Neuron 28: 585-594.

Csicsvari J, O’Neill J, Allen K, Senior T. 2007. Place-selective firing contributes to the reverse-order reactivation of CA1 
pyramidal cells during sharp waves in open-field exploration. Eur J Neurosci 26: 704-716.

Dabaghian Y, Mémoli F, Frank L, Carlsson G. 2012. A topological paradigm for hippocampal spatial map formation using persistent homology. PLoS Comput Biol 8: e1002581.

Dabaghian Y, Brandt VL, Frank LM. 2014. Reconceiving the hippocampal map as a topological template. Elife 3: e03476.

Davidson TJ, Kloosterman F, Wilson MA. 2009. Hippocampal replay of extended experience. Neuron 63: 497-507.

Davidson TJ, Anderson EB, Lerner TN, Ramakrishnan C, Mattias J, Grosenick LM, Kauvar IV, Frank LM, Deisseroth K. 2014. Subsecond cholinergic dynamics underlying hippocampal network state in freely-behaving rats. In Society for Neuroscience Abstracts, Program No. 566.18. 2014 Neuroscience Meeting Planner. Society for Neuroscience, Washington, DC. Online.

Diba K, Buzsáki G. 2007. Forward and reverse hippocampal place-cell sequences during ripples. Nat Neurosci 10: 1241 1242.

Dupret D, O’Neill J, Pleydell-Bouverie B, Csicsvari J. 2010. The reorganization and reactivation of hippocampal maps predict spatial memory performance. Nat Neurosci 13: 995-1002.

Eichenbaum H. 2000. Hippocampus: Mapping or memory? Curr Biol 10: R785-R787.

Eichenbaum H. 2013. What H.M. taught us. J Cogn Neurosci 25: $14-21$

Eichenbaum H, Cohen NJ. 2001. From conditioning to conscious recollection: Memory systems of the brain. Oxford University Press, New York.

Fanselow MS, Dong HW. 2010. Are the dorsal and ventral hippocampus functionally distinct structures? Neuron 65: 7-19.

Fenton AA, Csizmadia G, Muller RU. 2000a. Conjoint control of hippocampal place cell firing by two visual stimuli. I. The effects of moving the stimuli on firing field positions. J Gen Physiol 116: 191-209.

Fenton AA, Csizmadia G, Muller RU. 2000b. Conjoint control of hippocampal place cell firing by two visual stimuli. Ii. A vector-field theory that predicts modifications of the representation of the environment. J Gen Physiol 116: 211-221.

Foster DJ, Wilson MA. 2006. Reverse replay of behavioural sequences in hippocampal place cells during the awake state. Nature 440: 680-683.

Frank LM, Brown EN, Wilson MA. 2000. Trajectory encoding in the hippocampus and entorhinal cortex. Neuron 27: 169178 .

Girardeau G, Benchenane K, Wiener SI, Buzsáki G, Zugaro MB. 2009. Selective suppression of hippocampal ripples impairs spatial memory. Nat Neurosci 12: 1222-1223.

Jadhav SP, Kemere C, German PW, Frank LM. 2012. Awake hippocampal sharp-wave ripples support spatial memory. Science 336: 1454-1458.

Ji D, Wilson MA. 2007. Coordinated memory replay in the visual cortex and hippocampus during sleep. Nat Neurosci 10: $100-107$.

Karlsson MP, Frank LM. 2008. Network dynamics underlying the formation of sparse, informative representations in the hippocampus. J Neurosci 28: 14271-14281.

Karlsson MP, Frank LM. 2009. Awake replay of remote experiences in the hippocampus. Nat Neurosci 12: 913-918.

Kemere C, Carr MF, Karlsson MP, Frank LM. 2013. Rapid and continuous modulation of hippocampal network state during exploration of new places. PLOS ONE 8: e73114.

Kim SM, Frank LM. 2009. Hippocampal lesions impair rapid learning of a continuous spatial alternation task. PLOS ONE 4: e5494.

Kim SM, Ganguli S, Frank LM. 2012. Spatial information outflow from the hippocampal circuit: Distributed spatial coding and phase precession in the subiculum. $J$ Neurosci 32: $11539-11558$

Knight R. 1996. Contribution of human hippocampal region to novelty detection. Nature 383: 256-259.

Knight RT, Nakada T. 1998. Cortico-limbic circuits and novelty: A review of EEG and blood flow data. Rev Neurosci 9: 57-70.
Kudrimoti HS, Barnes CA, McNaughton BL. 1999. Reactivation of hippocampal cell assemblies: Effects of behavioral state, experience, and EEG dynamics. J Neurosci 19: 4090-4101.

Larkin MC, Lykken C, Tye LD, Wickelgren JG, Frank LM. 2014. Hippocampal output area CA1 broadcasts a generalized novelty signal during an object-place recognition task. Hippocampus 24: 773-783.

Lee AK, Wilson MA. 2002. Memory of sequential experience in the hippocampus during slow wave sleep. Neuron 36: $1183-$ 1194.

Lisman JE, Otmakhova NA. 2001. Storage, recall, and novelty detection of sequences by the hippocampus: Elaborating on the SOCRATIC model to account for normal and aberrant effects of dopamine. Hippocampus 11: 551-568.

Marr D. 1971. Simple memory: A theory for archicortex. Philos Trans R Soc Lond B Biol Sci 262: 23-81.

McClelland JL, McNaughton BL, O'Reilly RC. 1995. Why there are complementary learning systems in the hippocampus and neocortex: Insights from the successes and failures of connectionist models of learning and memory. Psychol Rev 102: $419-457$

Montgomery SM, Buzsáki G. 2007. y Oscillations dynamically couple hippocampal CA3 and CA1 regions during memory task performance. Proc Natl Acad Sci 104: 14495-14500.

Nadasdy Z, Hirase H, Czurkó A, Csicsvari J, Buzsáki G. 1999. Replay and time compression of recurring spike sequences in the hippocampus. J Neurosci 19: 9497-9507.

Nakashiba T, Buhl DL, McHugh TJ, Tonegawa S. 2009. Hippocampal CA3 output is crucial for ripple-associated reactivation and consolidation of memory. Neuron 62: 781-787.

Norimoto H, Mizunuma M, Ishikawa D, Matsuki N, Ikegaya Y. 2012. Muscarinic receptor activation disrupts hippocampal sharp wave-ripples. Brain Res 1461: 1-9.

O'Keefe J, Burgess N. 1996. Geometric determinants of the place fields of hippocampal neurons. Nature 381: 425-428.

O'Keefe J, Dostrovsky J. 1971. The hippocampus as a spatial map. Preliminary evidence from unit activity in the freelymoving rat. Brain Res 34: 171-175.

O’Neill J, Senior TJ, Allen K, Huxter JR, Csicsvari J. 2008. Reactivation of experience-dependent cell assembly patterns in the hippocampus. Nat Neurosci 11: 209-215.

O’Neill J, Pleydell-Bouverie B, Dupret D, Csicsvari J. 2010. Play it again: Reactivation of waking experience and memory. Trends Neurosci 33: 220-229.

O'Reilly RC, Norman KA. 2002. Hippocampal and neocortical contributions to memory: Advances in the complementary learning systems framework. Trends Cogn Sci 6: 505-510.

Pavlides C, Winson J. 1989. Influences of hippocampal place cell firing in the awake state on the activity of these cells during subsequent sleep episodes. J Neurosci 9: $2907-$ 2918.

Pfeiffer BE, Foster DJ. 2013. Hippocampal place-cell sequences depict future paths to remembered goals. Nature 497: 74-79.

Scoville WB, Milner B. 1957. Loss of recent memory after bilateral hippocampal lesions. J Neurol Neurosurg Psychiatry 20: $11-21$

Singer AC, Frank LM. 2009. Rewarded outcomes enhance reactivation of experience in the hippocampus. Neuron 64: 910-921

Singer AC, Karlsson MP, Nathe AR, Carr MF, Frank LM. 2010. Experience-dependent development of coordinated hippocampal spatial activity representing the similarity of related locations. J Neurosci 30: 11586-11604.

Singer AC, Carr MF, Karlsson MP, Frank LM. 2013. Hippocampal SWR activity predicts correct decisions during the initial learning of an alternation task. Neuron 77: 1163-1173.

Squire LR, Bayley PJ. 2007. The neuroscience of remote memory. Curr Opin Neurobiol 17: 185-196.

Sutherland GR, McNaughton B. 2000. Memory trace reactivation in hippocampal and neocortical neuronal ensembles. Curr Opin Neurobiol 10: 180-186.

Vandecasteele M, Varga V, Berényi A, Papp E, Barthó P, Venance L, Freund TF, Buzsáki G. 2014. Optogenetic activation 


\section{ANDERSON ET AL.}

of septal cholinergic neurons suppresses sharp wave ripples and enhances $\theta$ oscillations in the hippocampus. Proc Natl Acad Sci 111: 13535-13540.

Wilson MA, McNaughton BL. 1994. Reactivation of hippocampal ensemble memories during sleep. Science 265: 676-679.
Yang S, Yang S, Moreira T, Hoffman G, Carlson GC, Bender KJ, Alger BE, Tang CM. 2014. Interlamellar CA1 network in the hippocampus. Proc Natl Acad Sci 111: 12919-12924.

Yu JY, Frank LM. 2014. Hippocampal-cortical interaction in decision making. Neurobiol Learn Mem 117: 34-41. 


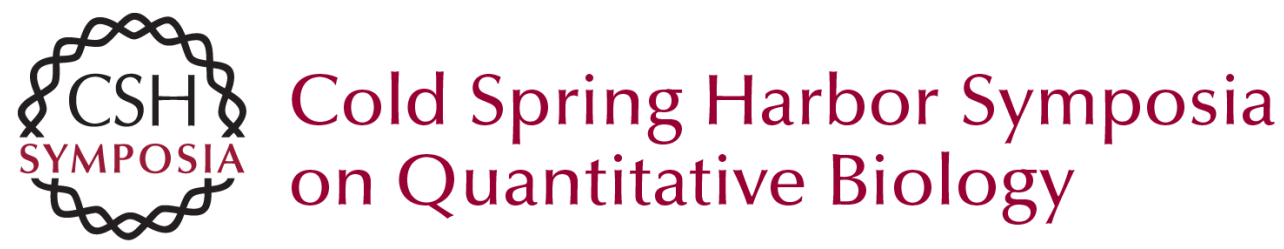

\section{Dynamic Hippocampal Circuits Support Learning- and Memory-Guided Behaviors}

Emily B. Anderson, Irene Grossrubatscher and Loren Frank

Cold Spring Harb Symp Quant Biol 2014 79: 51-58 originally published online April 28, 2015 Access the most recent version at doi:10.1101/sqb.2014.79.024760

References This article cites 61 articles, 15 of which can be accessed free at: http://symposium.cshlp.org/content/79/51.full.html\#ref-list-1

License

Email Alerting Receive free email alerts when new articles cite this article - sign up in Service the box at the top right corner of the article or click here. 